\title{
A Portable Device of Life Cycle Information Management
}

\author{
Zhang Weixin", Ruan Xiaoyan", Li Zhuolun', Liu Yia \\ Missile Department of University, Army Engineering University of PLA University, Wuhan, China \\ *ivanzwx@163.com, ${ }^{a .55026020 @ q q . c o m, ~}{ }^{b}$. lizhuolun1988@126.com
}

Keywords: FPGA, image recognition, video matrix, information managment

\begin{abstract}
This paper made an in-depth research of the weapon management with the method of intelligentization, which is based on image recognition, database and video matrix technology, designed the hardware circuit and software program, designed hardware circuit platform around the Cortex-A8 core processor in hardware, which was exclusively for the camera driver, image capture and recognition, prorammed image recognition and program of video matrix code with QT, and explained user management module, information acquisition module, status query module, and data communication module in detail. Results show that the system has a high identification rate correctly and a good performance in status query and weapon management.
\end{abstract}

\section{Introduction}

In the process of accelerating information weapon to be fielded in the forces on a large scale, the base unit must survey regularly the quantity and quality of equipment, as well as different levels of maintenance for the equipments and the components and special supporting tools, according to the equipment maintenance system requirements. The numerous vehicle and components and auxiliary maintenance tools of weapon system make work heavily and management inefficiently, when the information of the equipment states were checked traditionally eye by eye, heart by heart and hand by hand.

The information integrated management equipment based on the techniques of image recognition, database and cloud storage gathers the external image of the equipments and the components and the replacement parts by the image recognition system embedded in the portable terminal. The software could recognize, classify and offer the equipment states, such as the times of maintenance, the histories of service and the applications for abandoned assets, which provide decision support of the equipment life cycle management for the use personnel ${ }^{[1]}$.At the same time, each terminal provides the equipment information gathered by the USB protocol to the master computer, which provide real-time monitoring management and intelligent decisions support of the operation, maintenance, service and other devices belonged to superior equipment management department and intelligence department. 


\section{General Project}

The device is composed of a master computer and at least one terminal for the equipment of life cycle management, whose organizational structure of platform is shown in Figure 1. The master computer gathers the equipment information in the terminals and provide real-time monitoring management and maintenance decisions support, meanwhile provide data cleaning and system maintenance for the terminal and the addition or the lessen for the organizational structure and content of the life cycle management information.

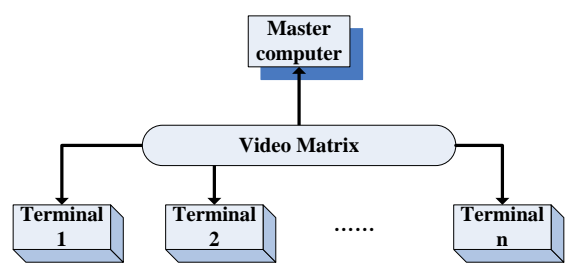

Fig. 1. The instructure of platform

\section{The Design of Hardware}

One terminal correspond one device which is a small portable device embedded Hd camera. The device structure is composed of the shell, Hd camera, embedded micro-host , interactive module and transmission interface and so on as shown in Figure 2.

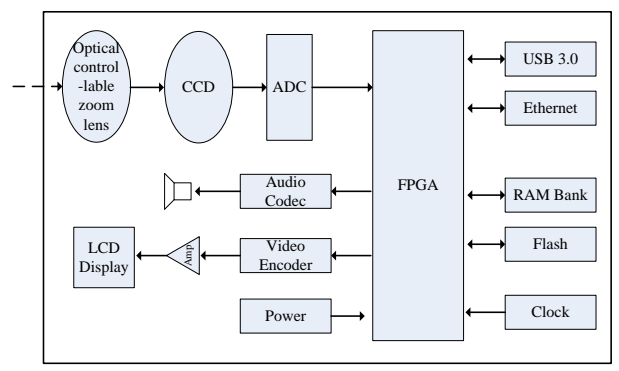

Fig. 2. Cnostructure of device

\subsection{The embedded micro-host}

The design of embedded micro-host includes the hardware and the software. The Cortex-A8 core processor is adopted by the hardware research ${ }^{[2]}$, and the corresponding peripheral circuit including storage system, peripheral interface, etc is designed. The software includes custom operating system, image recognition algorithm, and database management.

\subsection{Hd camera}

The Hd camera collected the appearance information of the device and the components is proposed with 3 million pixels, which can recognize images by the accuracy rate and the reliability.

\subsection{The reading control of 32-channel data of USB}

The reading control of 32-channel data of USB is mainly composed of hubs and TS3USB221E switch chips ${ }^{[3]}$. Each controlling hub is connected to 4 interface hub, and each interface hub is connected to 4 USB switches.Two interface are controlled by each USB switch, and it can realize 
the topology control of reading 32-channel USB data as shown in Figure $3^{[4]}$.

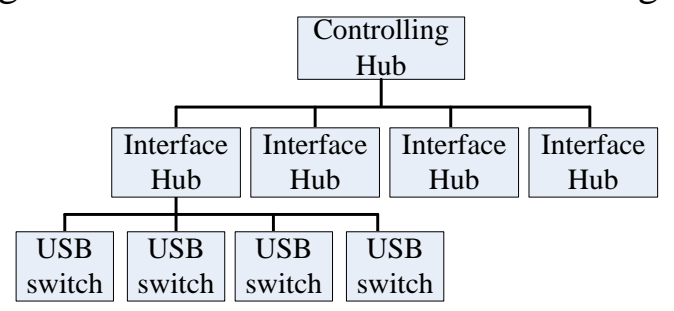

Fig. 3. Topology control of reading 32-channel USB data

Using the video matrix to transport the information, which is formed a large amount of transmission data after collection, is in favor of uploading all data automatically in order to solve this problem.

The distributed storage technology is adopted in transit, which use video information as redundant backup at the grass-roots level management and distributed store automatically the key information including the maintenance and the life cycle in the superior management system.

Single chip microcomputer core processing module, USB switch module, power supply module and circuit design make up the hardware of the system which is able to transmit signals with the maximum 32 channel signals.

\subsection{Power supply module}

System power requires 3.3V for single chip microcomputer, 1.2V for FPGA core voltage, 3.3V for PFAG driving voltage, 3.3V for high speed USB switch, 3.3V for control hub and $5 \mathrm{~V}$ for USB interface.

\subsection{Others}

The design of the shell is similar to the PAD in the market, which is easy to hold and has a certain shockproof. The interactive panel convenient, which was operated this terminal equipment for the users, uses 9.7-inch LCD for display panel. The resolution ratio of LCD is 1024x768.The peripheral interface is intended to choose the standard Micro USB port for the generality, which is convenient to channel the generated report status to other devices.

\section{The Development of Software}

LINUX is selected for the function of the management system as the application environment of the underlying development management system which includes the drives of the standard USB port, the Hd camera and the LCD. Qt as a development tool of the cross-platform C++ graphical interface is used to design human-computer interaction interface, which includes the initial background interface and the system startup interface.

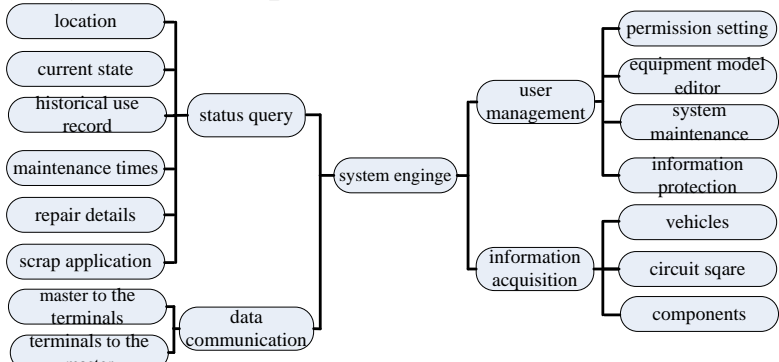

Fig. 4. The structure of the software 
The database structure of the manage system is quadrated to the user management module, information acquisition module, status query module and data communication module. The organization structure of the manage system is shown in Figure 4.

\subsection{The user management module}

The main composition of the module is the permission setting and the equipment model editor.

The former identifies the identity information of the using personnel for two core functions of the platform. One as a input device of the information management can set the user permission as an information entry so as to be the master control and the terminal dedicated. The other as a use device of the information management can provide the life cycle information.

The latter can realize data cleaning or system maintenance for the terminal and the addition or the lessen for the life cycle management information from different type of equipment, which means building all status options when the device requires gathering status information in the other words.

The two auxiliary functions of the user management are data cleaning or system maintenance for the terminal to control the terminal and information protection, in order to prevent copying the status information from the device by mean of the decoding software.

\subsection{The information acquisition module}

The module extracting the device information to the database completes the image recognition for the devices, the vehicles the components and the circuit square parts. It can be done at the same time that the selection and entry of the life cycle information of the device status, which include type, appellation, code name, quantity, status condition, location, maintenance times, contact details of the repair shops, scrap application, service times and so on.

\subsection{The status query modulle}

The module can rapid position according to the name/ type /code name/image, and complete the query for location, the current state, historical use record, maintenance times, contact details of the technical support from the equipment repair shops and the discarded age limit.

The equipment can provide the maintenance condition, maintenance times and decision support for the user and the manager when the system boots. The one result queried is shown in Figure 5.

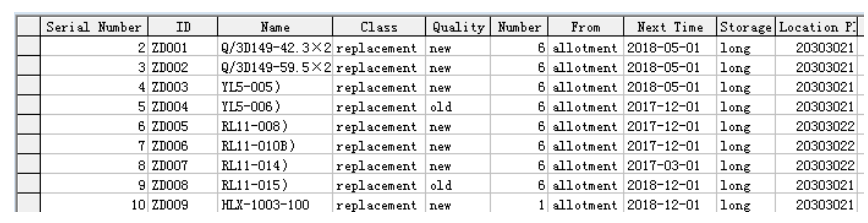

Fig. 5. The one result of query

\subsection{The data communication module}

The module completes data cleaning or system maintenance to control the terminal and data communication in the times of editing devices model gathered from the terminal. In the meantime it is done that the equipment information are summarized to the communication for the master. 


\subsection{The program of video matrix}

Program includes the redact for read state machine of the device, the design of the user interface, database access module and video-audio conversion module. The status of the read state machine are start, stop, open port, waiting device access, waiting for the decryption, waiting for equipment check, uploading data, equipment encryption and close port as shown Figure $6{ }^{[4]}$.The timed management mode is adopted in the status, what means opening in the last state, closing in the next state and returning to specified state if there is no response over time.

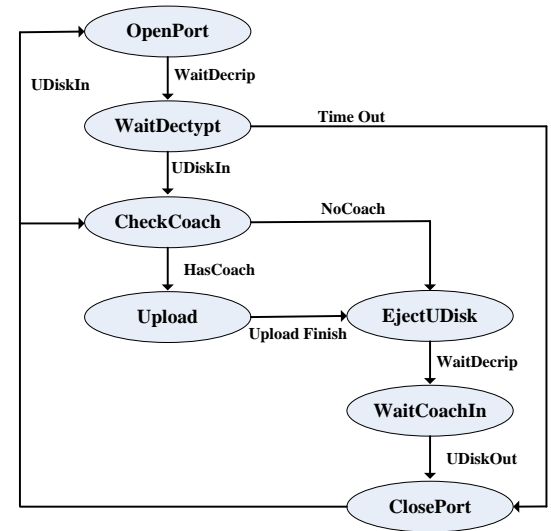

Fig. 6. Process of state machine

\section{Conclusion}

The equipment realized automatic management, not only provided query approach and decision support in real time for management, but also reduced manpower and material cost and improve management efficiency. Meanwhile, it had an in-depth research of transplant of platform.

\section{References}

[1] Yin Bin, Zheng Xianbin. Study of standardization under the background of weapons and equipment life-cycle management. Journal of Sichuan Ordnance, 2013, 10:63-66.

[2] Zhai Sheping, Li Wei, Ma Chuanbin. Image recognition of digital computing and FPGA implementation [J]. Information Technology, 2016, 3:15-18.

[3] Wang Zhibin.The portable system development of the image sampling based on USB and object recognition [D]. Journal of Shanghai Jiaotong University, 2008:39-42.

[4] Shi Dianyang. 32-port video matrix system design.Huazhong University of Science and Technology, 2013:16-19,4447. 